\title{
Radiative transition probabilities in the O-like sequence ${ }^{\star}$
}

\author{
E. Landi
}

\author{
Artep, Inc., Ellicott City, MD 21042, USA and Naval Research Laboratory, Washington DC, 20375-5320, USA \\ e-mail: landi@poppeo.nrl.navy.mil
}

Received 30 September 2004 / Accepted 13 December 2004

\begin{abstract}
In the present work a complete set of radiative transition rates is calculated for all for the O-like ions with $Z=11-30$. Energy levels, oscillator strengths and $A$ values are computed for all transitions within the $n=2$ complex and are compared with previous calculations, where available. Calculations are carried out using the Superstructure code. The present work provides for the first time a self-consistent, complete set of $A$ values necessary for the calculation of line emissivities and synthetic spectra for all the ions considered, filling several gaps in the existing literature. The present data are especially suited for the analysis of spectral lines emitted by the less-abundant elements in the universe, for which few if any data were available in the literature.
\end{abstract}

Key words. atomic data - atomic processes - radiation mechanisms: general - line: formation - Sun: corona

\section{Introduction}

Spectral lines emitted by ions belonging to the oxygen-like sequence have long been known in the literature for their diagnostic potential for astrophysical plasmas in a variety of different physical conditions. The importance of these ions is given by the rather prominent spectral lines that they generate from both allowed and forbidden transitions. Such lines have been long observed in all wavelength ranges. Allowed transitions within the $n=2$ complex give rise to prominent lines in the extreme-ultraviolet spectrum, that have been used over the years for plasma diagnostics in solar and stellar plasmas in all conditions; forbidden transitions within the ground configuration levels give rise to a number of lines in the ultraviolet, visible and infrared ranges which have been observed in solar, stellar and in planetary nebulae spectra. Recently, allowed transitions from excited $n=3$ configurations from several O-like ions have been observed in solar ultraviolet spectra (Kink et al. 1997; Kink \& Engström 1997) and have been found to provide excellent temperature diagnostic tools when used in combination with forbidden lines within the ground configuration, usually found in their proximity. Moreover, lines from O-like ions from less abundant elements, such as $\mathrm{P}, \mathrm{Cl}, \mathrm{K}, \mathrm{Ti}, \mathrm{Cr}, \mathrm{Mn}$ and $\mathrm{Co}$, have been identified in solar spectra (Feldman et al. 2000; Feldman et al. 2004); these ions allow more accurate studies of the chemical composition of the observed plasmas.

The O-like isoelectronic sequence has been paid little attention in the past as far as electron excitation is concerned, but

* Tables 3-8 are also available in electronic form at the CDS via anonymous ftp to cdsarc.u-strasbg.fr (130.79.128.5) or via http: //cdsweb.u-strasbg.fr/cgi-bin/qcat?J/A+A/434/365 several authors studied O-like systems in an effort to determine the energies of their levels, their radiative rates and lifetimes. A few complete calculations of radiative data that encompass the whole isoelectronic sequence, with the exceptions of the very low stages of ionization, have been carried out by Cheng et al. (1979), Fawcett (1986) and Zhang \& Sampson (2002). However, the latter two calculations only provided data for allowed transitions between the ground and the excited configurations, and do not allow to calculate level populations and line intensities. Cheng et al. (1979) included the three configurations in the $n=2$ complex in their atomic model, and published a complete dataset of radiative data. Another complete dataset was provided by Froese Fischer \& Tachiev (2004) and Tachiev \& Froese Fischer (2002), but limited to the O-like ions with $Z \leq 20$. Galavis et al. (1997) provided radiative transition probabilities for forbidden transitions within the ground configuration of the O-like sequence up to Ni. A similar calculation was carried out by Froese Fischer \& Saha (1983), Baluja \& Zeippen (1988) and Vilkas et al. (1994): the latter two datasets however considered only the most abundant elements up to Ni. Calculations limited to a few ions between $Z=26$ and $Z=36$ have been reported by Feldman et al. (1985); Bhatia et al. (1979) published data for Si VII, S IX and Ar XI only, while Bhatia et al. (1980) and Bhatia (1982) considered only O-like Ti and Mn, respectively. These calculations were carried out mostly using a limited number of configurations in the atomic model. Recently, Bhatia et al. (2003a,b) and Bhatia \& Landi (2003a,b) published complete data for a few individual ions (Ne III, Si VII, S IX and Ni XXI), while Lolergue et al. (1985) and Butler \& Zeippen (2001) provided a complete dataset for Fe XIX. In general, all the less abundant ions in the 
O-like sequence with $20 \leq Z \leq 30$ have incomplete datasets, or their data have been calculated using limited atomic models. However, databases such as CHIANTI (Dere et al. 1997; Young et al. 2003), require dataset as complete, accurate and self-consistent as possible, in order to provide the astrophysical community with tools and data to carry out accurate plasma diagnostics. Of particular importance for these databases are complete sets of accurate $A$ values and oscillator strengths.

The aim of the present work is to calculate a complete set of radiative transition probabilites ( $A$ values and oscillator strengths) for all possible $n=2$ transitions in the O-like sequence for all ions with $11 \leq Z \leq 30$, in an effort to provide a complete, self-consistent and accurate dataset; we are mainly interested in providing a complete set of data for the less abundant elements in the O-like sequence ( $\mathrm{P}, \mathrm{Cl}, \mathrm{K}, \mathrm{Ti}, \mathrm{Cr}, \mathrm{Mn}, \mathrm{Co}$ ), for the first time. This will be done by using a comprehensive atomic model that includes all the relevant configurations in the O-like sequence, which is described in Sect. 2. The present results are compared with previous calculations, in order to assess their accuracy, in Sect. 3. Section 4 summarizes the results.

\section{Atomic data}

The atomic data have been calculated using computer programs originally developed at University College London. These programs have been updated over the years. The energy levels, oscillator strengths, and radiative transition rates have been calculated by using the Superstructure program described by Eissner et al. (1974). The configurations we are interested in are $2 s^{2} 2 p^{4}$, $2 s 2 p^{5}$ and $2 p^{6}$, giving rise to 10 fine-structure levels. In order to calculate accurate atomic level energies and radiative transition rates an atomic model including 24 configurations has been used. The configurations are listed below.

$$
\begin{array}{ll}
2 \mathrm{~s}^{2} 2 \mathrm{p}^{4} & \\
2 \mathrm{~s}^{5} \mathrm{p}^{5} & \\
2 \mathrm{p}^{6} & \\
2 \mathrm{~s}^{2} 2 \mathrm{p}^{3} 3 l & l=\mathrm{s}, \mathrm{p}, \mathrm{d} \\
2 \mathrm{~s} 2 \mathrm{p}^{4} 3 l & l=\mathrm{s}, \mathrm{d}, \mathrm{d} \\
2 \mathrm{p}^{5} 3 l & l=\mathrm{s}, \mathrm{p}, \mathrm{d} \\
& \\
2 \mathrm{~s}^{2} 2 \mathrm{p}^{3} 4 l & l=\mathrm{s}, \mathrm{p}, \mathrm{d}, \mathrm{f} \\
2 \mathrm{~s}^{4} \mathrm{p}^{4} 4 l & l=\mathrm{s}, \mathrm{p}, \mathrm{d}, \mathrm{f} \\
2 \mathrm{p}^{5} 4 l & l=\mathrm{s}, \mathrm{p}, \mathrm{d}, \mathrm{f} .
\end{array}
$$

The wavefunctions are of configuration interaction type and each configuration is expanded in terms of Slater orbitals. The radial functions are calculated in a scaled Thomas-Fermi-Amaldi potential. The potential depends upon parameters $\lambda_{n l}$ which are determined variationally by optimizing the weighted sum of the term energies. The relativistic corrections are included by using the Breit-Pauli Hamiltonian as a perturbation to the nonrelativistic Hamiltonian. The Breit-Pauli Hamiltonian adopted in Superstructure includes mass variation, Darwin and spin-orbit operators, as well as spin-other orbit, mutual spin-orbit and spin-spin operators; more details can be found in Eissner et al. (1974). Energy levels, oscillator strengths, and radiative transition rates are calculated in intermediate coupling. The calculated energies are listed in Table 1 along with experimentally determined energies from the Edlen (1983); experimental energies for Fe XIX and Ni XXI come from Shirai et al. (2000). We use the calculated energies and the wave functions to calculate all the radiative data; oscillator strengths and $A$ values are then corrected in order to take into account laboratory wavelengths. The resulting corrected $A$ values and weighted oscillator strengths (obtained multiplying the corrected absorption oscillator strength for the statistical weight of the lower level of each transition) are given in Tables 3 to 8 .

\section{Comparison with earlier work}

In the past, several different calculations have been carried out on O-like ions, some involving one or only a few ions, some providing datasets across the whole O-like sequence. In order to assess the accuracy of the present calculations, we have compared them with results found in the literature. The availability of many independent calculations carried out using different theoretical methods and approximations helps in assessing the accuracy of our results. Some of these calculations were carried out in the 1980s, when computers had a more limited computational capabilities, so atomic models had to be restricted to only a handful of configurations, at the expense of accuracy in the computed wavefunctions. With modern computers the situation is greatly improved and the set of configurations that now can be included is much larger. This allows to take into account with greater accuracy the interactions between different configurations and improve the results. Therefore, in our comparison, we will focus mostly on recent, sophisticated calculations, where available; we will also consider the comprehensive computations by Chang et al. (1979) and Froese Fischer \& Saha (1983), given the accuracy of the approximations adopted in these works.

Table 2 compares energy levels obtained in the present calculations (ab initio results from the 24-configuration model) with the observed ones, and with those obtained by other calculations: Cheng et al. (1979), Tachiev \& Froese Fischer (2002), Vilkas et al. (1994) and Zhang \& Sampson (2002): all these works were carried out using different methods and approximations than Superstructure. The comparison is shown for Si VII, Ca XIII and Fe XIX. Results show that for Fe XIX all calculations have comparable accuracy and reproduce the observed energies within $1 \%$; all calculations provide similar results. At low- $Z$, the comparison for Si VII shows that results from the more elaborate models from Tachiev \& Froese Fischer (2002) and Vilkas et al. (1994) provide closer agreement with observations than those from Cheng et al. (1979) and the present work. The latter two calculations provide similar results for the excited configurations, while Cheng et al. (1979) has closer agreement with observations for the ground configuration than the present calculation. Results for Ca XIII show that the present calculation is better at reproducing observations than for Si VII, and differences from theother calculations are smaller than at lower $Z$. However, Tachiev \& Froese Fischer (2002) and Vilkas et al. (1994) still have better agreement with observed energies. This seems to indicate that the approximations in Superstructure are 
Table 1. Energies for the $n=2$ levels in the O-like isoelectronic sequence. The first entry for each level is the experimental value, the second entry is the theoretical value calculated in the present work. Experimental energies come from Edlen (1983), with the only exception of Fe XIX and Ni XXI, which are taken from Shirai et al. (2000). Energies are in $\mathrm{cm}^{-1}$.

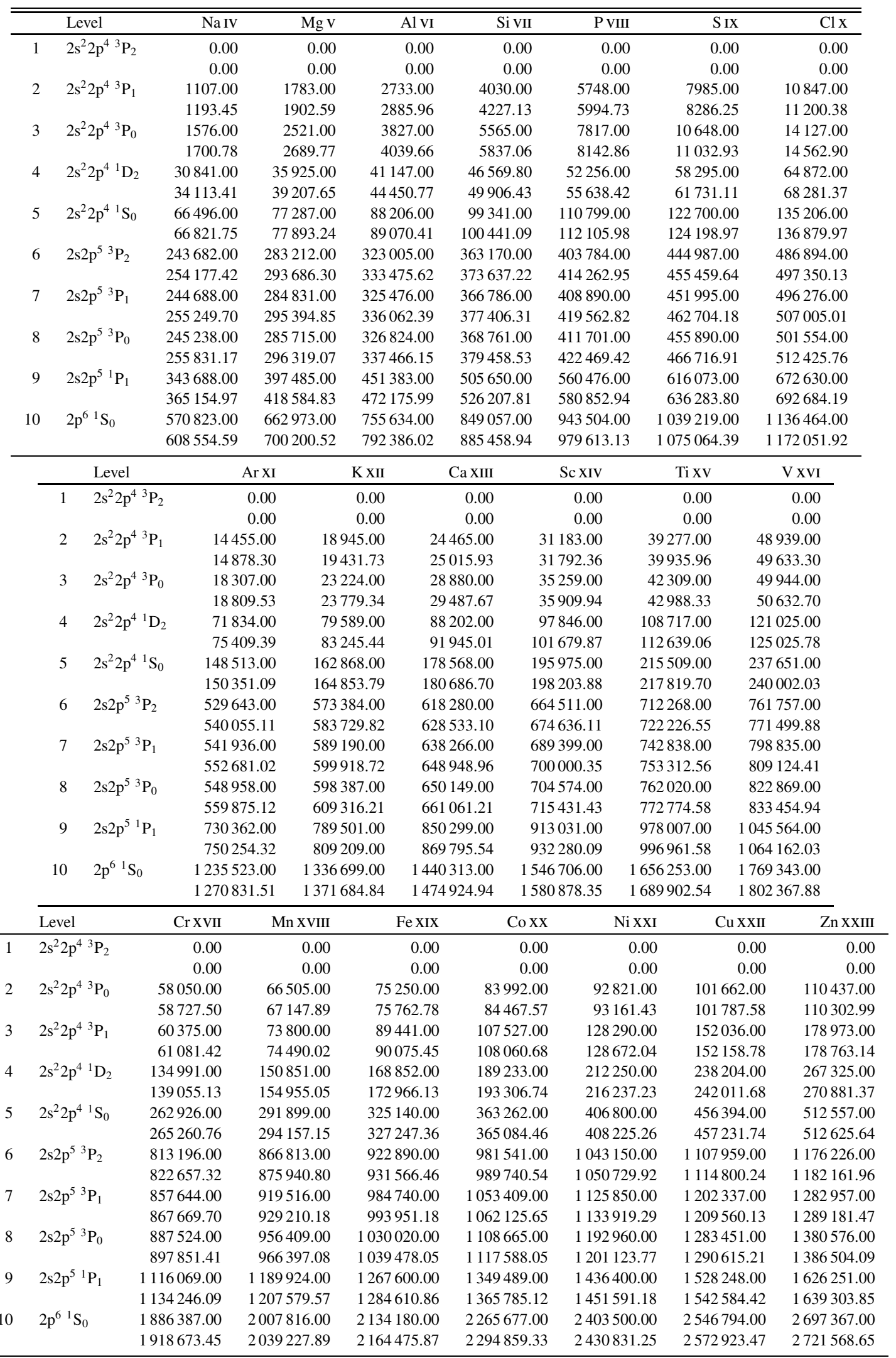


Table 2. Comparison of observed and computed energy levels in the O-like sequence. Only the cases of Si VII, Ca XIII and Fe XIX are reported. $E_{\mathrm{obs}}$ : observed energy level, from Edlen (1983 - Si VII, Ca XIII) and Shirai et al. (2000 - Fe XIX); 24C: present work, 24-configuration calculation; C79: Cheng et al. (1979); TFF02: Tachiev \& Froese Fischer (2002); V94: Vilkas et al. (1994); ZS02: Zhang \& Sampson (2002).

\begin{tabular}{|c|c|c|c|c|c|}
\hline \multicolumn{6}{|c|}{ Si VII } \\
\hline Level & $E_{\text {obs }}$ & $24 \mathrm{C}$ & C79 & TFF02 & V94 \\
\hline 1 & 0 & 0 & 0 & 0 & 0 \\
\hline 2 & 4030 & 4227 & 3997 & 4034 & 4017 \\
\hline 3 & 5565 & 5837 & 5456 & 5546 & 5566 \\
\hline 4 & 46570 & 49906 & 48693 & 46910 & 46520 \\
\hline 5 & 99341 & 100441 & 95618 & 99315 & 99134 \\
\hline 6 & 363170 & 373637 & 372074 & 364085 & 362832 \\
\hline 7 & 366786 & 377406 & 375669 & 367698 & 364880 \\
\hline 8 & 368761 & 379459 & 377559 & 369675 & 366849 \\
\hline 9 & 505650 & 526208 & 527122 & 507551 & 504125 \\
\hline 10 & 849507 & 885459 & 888769 & 852002 & 844169 \\
\hline \multicolumn{6}{|c|}{ Ca XIII } \\
\hline Level & $E_{\mathrm{obs}}$ & $24 \mathrm{C}$ & C79 & TFF02 & V94 \\
\hline 1 & 0 & 0 & 0 & 0 & 0 \\
\hline 2 & 24465 & 25016 & 24646 & 24800 & 24209 \\
\hline 3 & 28880 & 29488 & 28857 & 28832 & 28737 \\
\hline 4 & 88202 & 91045 & 91046 & 89087 & 88287 \\
\hline 5 & 178568 & 180687 & 174355 & 178643 & 178016 \\
\hline 6 & 618280 & 628533 & 627311 & 621713 & 616067 \\
\hline 7 & 638266 & 648949 & 647961 & 641939 & 635927 \\
\hline 8 & 650149 & 661061 & 659795 & 653989 & 647728 \\
\hline 9 & 850299 & 869796 & 872522 & 854658 & 847229 \\
\hline 10 & 1440313 & 1474925 & 1480522 & 1445483 & 1434221 \\
\hline \multicolumn{6}{|c|}{ Fe XIX } \\
\hline Level & $E_{\text {obs }}$ & $24 \mathrm{C}$ & C79 & $\mathrm{ZS} 02$ & V94 \\
\hline 1 & 0 & 0 & 0 & 0 & 0 \\
\hline 2 & 75250 & 75762 & 76016 & 74160 & 74712 \\
\hline 3 & 89441 & 90075 & 89003 & 89129 & 87559 \\
\hline 4 & 168852 & 172966 & 170557 & 170422 & 167882 \\
\hline 5 & 325140 & 327247 & 322602 & 321201 & 321124 \\
\hline 6 & 922890 & 931566 & 930851 & 930902 & 917431 \\
\hline 7 & 984740 & 993951 & 993068 & 993123 & 978242 \\
\hline 8 & 1030020 & 1039478 & 1037725 & 1037786 & 1022750 \\
\hline 9 & 1267600 & 1284611 & 1287937 & 1289413 & 1258927 \\
\hline 10 & 2134180 & 2164476 & 2172572 & 2176091 & 2120179 \\
\hline
\end{tabular}

less accurate in calculating ab initio energy levels for lower stages of ionization than the other methods.

To check how this more limited accuracy is propagated to the radiative transition rates, which are the main focus of the present work, we have carried out extensive comparisons of $A$ values in the present work with those from other authors, with special emphasys on the lower stages of ionization.

The forbidden transitions within the ground configurations are very important both for level population calculation and because they provide some strong lines observed in astrophysical spectra. We have compared our results with those obtained by Galavis et al. (1997 - G97), Baluja \& Zeippen (1988 - BZ88), Tachiev \& Froese Fischer (2002 - TFF02), Froese Fischer \& Saha (1983 - FS83), and Cheng et al. (1979-C79). Agreement within $10 \%$ is found for all transitions in all ions except for the results from Cheng et al. (1979), who show differences from all other calculations by $10 \%$ to $30 \%$. Transitions involving
$\Delta J=2$ and the ${ }^{3} \mathrm{P}$ ground term $\left({ }^{3} \mathrm{P}_{0}-{ }^{1} \mathrm{D}_{2}\right.$ and $\left.{ }^{3} \mathrm{P}_{2}-{ }^{1} \mathrm{~S}_{0}\right)$ show a larger discrepancy. An example is given in Table 9, where a detailed comparison is shown for Si VII. The $A$ value of the ${ }^{3} \mathrm{P}_{0}-{ }^{1} \mathrm{D}_{2}$ transition is higher in the present calculation by $20 \%$ : this disagreement decreases with $\mathrm{Z}$, and the different calculations agree within $10 \%$ for all ions heavier than K XII. Problems with the ${ }^{3} \mathrm{P}_{2}-{ }^{1} \mathrm{~S}_{0}$ transition are persistent in all ions and cause the various calculations to disagree up to $30 \%$. However, both transitions (electric quadrupole) have very limited influence on the level populations and do not give rise to any observed lines. An example of the disagreement is shown in Table 9, where a comparison between the results from the present work (24C) and other sources are presented for Si VII. For comparison purposes, we have also included the results (6C) obtained with the same codes and approximations of the present calculations, but adopting a more limited atomic model, that includes the six lowest configurations only: this model, more limited than all 
Table 3. $A$ values for the O-like sequence (Na IV to S IX). E1: electric dipole transitions; E1f: spin-forbidden E1 transitions; E2: electric quadrupole transitions; M1: magnetic dipole transitions; M2: magnetic quadrupole transitions.

\begin{tabular}{|c|c|c|c|c|c|c|c|c|}
\hline Down & Up & Type & $\mathrm{Na} \mathrm{IV}$ & $\mathrm{Mg} \mathrm{V}$ & $\mathrm{Al} \mathrm{VI}$ & Si VII & P VIII & S IX \\
\hline $1-$ & 2 & M1 & $3.048-02$ & $1.273-01$ & $4.575-01$ & $1.467+00$ & $4.249+00$ & $1.136+01$ \\
\hline $1-$ & 3 & E2 & $1.820-07$ & $1.062-06$ & 5.199-06 & $2.172-05$ & 8.034-05 & $2.648-04$ \\
\hline $1-$ & 4 & M1 & $5.925-01$ & $1.798+00$ & $4.863+00$ & $1.186+01$ & $2.688+01$ & $5.720+01$ \\
\hline $1-$ & 5 & E2 & $8.670-03$ & $2.049-02$ & $4.419-02$ & $8.592-02$ & $1.571-01$ & 2.703-01 \\
\hline 1 - & 6 & E1 & $6.077+09$ & $7.364+09$ & $8.740+09$ & $1.019+10$ & $1.172+10$ & $1.332+10$ \\
\hline $1-$ & 7 & E1 & $3.424+09$ & $4.172+09$ & $4.985+09$ & $5.866+09$ & $6.815+09$ & $7.845+09$ \\
\hline $1-$ & 8 & M2 & $2.314+00$ & $3.792+00$ & $5.753+00$ & $8.267+00$ & $1.134+01$ & $1.508+01$ \\
\hline $1-$ & 9 & E1f & $1.479+07$ & $3.285+07$ & $6.812+07$ & $1.308+08$ & $2.364+08$ & $4.043+08$ \\
\hline $1-$ & 10 & E2 & $1.502+02$ & $3.172+02$ & $6.284+02$ & $1.136+03$ & $1.951+03$ & $3.195+03$ \\
\hline $2-$ & 3 & M1 & $5.562-03$ & $2.166-02$ & $7.108-02$ & $1.945-01$ & 4.753-01 & $1.010+00$ \\
\hline $2-$ & 4 & M1 & $1.769-01$ & 5.146-01 & $1.321+00$ & $3.021+00$ & $6.345+00$ & $1.234+01$ \\
\hline $2-$ & 5 & M1 & $8.261+00$ & $2.417+01$ & $6.336+01$ & $1.509+02$ & $3.339+02$ & $6.940+02$ \\
\hline $2-$ & 6 & E1 & $1.997+09$ & $2.408+09$ & $2.840+09$ & $3.290+09$ & $3.752+09$ & $4.225+09$ \\
\hline $2-$ & 7 & E1 & $2.024+09$ & $2.451+09$ & $2.909+09$ & $3.394+09$ & $3.902+09$ & $4.436+09$ \\
\hline $2-$ & 8 & E1 & $8.154+09$ & $9.911+09$ & $1.180+10$ & $1.381+10$ & $1.597+10$ & $1.825+10$ \\
\hline $2-$ & 9 & E1f & $3.223+05$ & $7.129+05$ & $1.529+06$ & $3.104+06$ & $5.923+06$ & $1.077+07$ \\
\hline $2-$ & 10 & M1 & $2.465+00$ & $6.233+00$ & $1.580+01$ & $3.647+01$ & $7.856+01$ & $1.590+02$ \\
\hline $3-$ & 4 & E2 & $3.661-05$ & $7.762-05$ & $1.534-04$ & 2.806-04 & $4.875-04$ & 8.079-04 \\
\hline $3-$ & 6 & M2 & 4.998-01 & $8.425-01$ & $1.325+00$ & $1.982+00$ & $2.870+00$ & $4.042+00$ \\
\hline $3-$ & 7 & E1 & $2.680+09$ & $3.240+09$ & $3.834+09$ & $4.457+09$ & $5.105+09$ & $5.778+09$ \\
\hline $3-$ & 9 & E1f & $4.582+05$ & $1.382+06$ & $3.200+06$ & $6.618+06$ & $1.273+07$ & $2.318+07$ \\
\hline $4-$ & 5 & E2 & $3.600+00$ & $4.329+00$ & $5.080+00$ & $5.876+00$ & $6.712+00$ & $7.610+00$ \\
\hline $4-$ & 6 & E1f & $1.944+06$ & $4.558+06$ & $9.773+06$ & $1.923+07$ & $3.553+07$ & $6.198+07$ \\
\hline $4-$ & 7 & E1f & $1.642+05$ & $3.496+05$ & $6.840+05$ & $1.225+06$ & $2.037+06$ & $3.148+06$ \\
\hline $4-$ & 8 & M2 & $2.274+00$ & $3.897+00$ & $6.228+00$ & $9.490+00$ & $1.394+01$ & $1.994+01$ \\
\hline $4-$ & 9 & E1 & $2.934+10$ & $3.507+10$ & $4.103+10$ & $4.720+10$ & $5.353+10$ & $6.005+10$ \\
\hline $4-$ & 10 & E2 & $1.781+05$ & $2.481+05$ & $2.753+05$ & $2.993+05$ & $3.219+05$ & $3.438+05$ \\
\hline $5-$ & 6 & M2 & $3.092-01$ & $4.939-01$ & 7.384-01 & $1.049+00$ & $1.426+00$ & $1.870+00$ \\
\hline $5-$ & 7 & E1f & $3.819+05$ & $8.450+05$ & $1.776+06$ & $3.504+06$ & $6.619+06$ & $1.199+07$ \\
\hline $5-$ & 9 & E1 & $1.423+09$ & $1.980+09$ & $2.498+09$ & $3.010+09$ & $3.531+09$ & $4.062+09$ \\
\hline $6-$ & 7 & M1 & $2.288-02$ & $9.538-02$ & $3.374-01$ & $1.062+00$ & $2.990+00$ & $7.729+00$ \\
\hline $6-$ & 8 & E2 & $1.666-07$ & $9.856-07$ & 4.864-06 & $2.114-05$ & $8.105-05$ & $2.808-04$ \\
\hline $6-$ & 9 & M1 & $1.072+00$ & $3.250+00$ & $8.711+00$ & $2.124+01$ & $4.793+01$ & $1.016+02$ \\
\hline $6-$ & 10 & M2 & $4.894+01$ & $7.999+01$ & $1.227+02$ & $1.789+02$ & $2.513+02$ & $3.429+02$ \\
\hline $7-$ & 8 & M1 & $8.971-03$ & $3.726-02$ & $1.315-01$ & 4.154-01 & $1.198+00$ & $3.184+00$ \\
\hline $7-$ & 9 & M1 & $6.403-01$ & $1.906+00$ & $5.004+00$ & $1.194+01$ & $2.628+01$ & $5.418+01$ \\
\hline $7-$ & 10 & E1f & $2.800+06$ & $7.519+06$ & $1.677+07$ & $3.473+07$ & $6.751+07$ & $1.243+08$ \\
\hline $8-$ & 9 & M1 & $8.136-01$ & $2.424+00$ & $6.347+00$ & $1.504+01$ & $3.277+01$ & $6.666+01$ \\
\hline $9-$ & 10 & E1 & $2.065+10$ & $2.603+10$ & $3.200+10$ & $3.829+10$ & $4.490+10$ & $5.181+10$ \\
\hline
\end{tabular}

the others, simulates the models adopted in many early calculations. The differences rise to $20 \%$ in all transitions, except those with $\Delta J=2$, where differences range from $30 \%$ to a factor of 2 .

Table 10 shows the comparison between several different calculations of allowed (E1) and spin-forbidden E1 transitions between the ground and the $2 \mathrm{~s} 2 \mathrm{p}^{5}$ configurations, and the $2 \mathrm{~s} 2 \mathrm{p}^{5}$ and the $2 \mathrm{p}^{6}$ configurations for S IX. Table 10 also reports the data published by Bhatia \& Landi (2003b), calculated using a 6-configuration atomic model (6C): these $A$ values are usually higher than those from the present calculation by $10 \%$ to $40 \%$, a clear indication that a limited number of 
Table 4. Weighted oscillator strengths for the O-like sequence (Na IV to S IX). E1: electric dipole transitions; E1f: spin-forbidden E1 transitions; E2: electric quadrupole transitions; M1: magnetic dipole transitions; M2: magnetic quadrupole transitions.

\begin{tabular}{|c|c|c|c|c|c|c|c|c|}
\hline Down & Up & Type & $\mathrm{Na} I V$ & $\operatorname{Mg~V}$ & $\mathrm{Al}$ VI & Si VII & P VIII & S IX \\
\hline 1 - & 2 & M1 & $0.000+00$ & $0.000+00$ & $0.000+00$ & $0.000+00$ & $0.000+00$ & $0.000+00$ \\
\hline 1 - & 3 & E2 & $0.000+00$ & $0.000+00$ & $0.000+00$ & $0.000+00$ & $0.000+00$ & $0.000+00$ \\
\hline $1-$ & 4 & M1 & $0.000+00$ & $0.000+00$ & $0.000+00$ & $0.000+00$ & $0.000+00$ & $0.000+00$ \\
\hline $1-$ & 5 & E2 & $0.000+00$ & $0.000+00$ & $0.000+00$ & $0.000+00$ & $0.000+00$ & $0.000+00$ \\
\hline $1-$ & 6 & E1 & 7.675-01 & $6.886-01$ & $6.283-01$ & $5.796-01$ & 5.393-01 & $5.044-01$ \\
\hline $1-$ & 7 & E1 & $2.574-01$ & $2.314-01$ & $2.118-01$ & $1.962-01$ & $1.834-01$ & $1.728-01$ \\
\hline $1-$ & 8 & M2 & $0.000+00$ & $0.000+00$ & $0.000+00$ & $0.000+00$ & $0.000+00$ & $0.000+00$ \\
\hline $1-$ & 9 & E1f & $5.635-04$ & $9.355-04$ & $1.504-03$ & $2.302-03$ & $3.386-03$ & $4.793-03$ \\
\hline $1-$ & 10 & E2 & $0.000+00$ & $0.000+00$ & $0.000+00$ & $0.000+00$ & $0.000+00$ & $0.000+00$ \\
\hline $2-$ & 3 & M1 & $0.000+00$ & $0.000+00$ & $0.000+00$ & $0.000+00$ & $0.000+00$ & $0.000+00$ \\
\hline $2-$ & 4 & M1 & $0.000+00$ & $0.000+00$ & $0.000+00$ & $0.000+00$ & $0.000+00$ & $0.000+00$ \\
\hline $2-$ & 5 & M1 & $0.000+00$ & $0.000+00$ & $0.000+00$ & $0.000+00$ & $0.000+00$ & $0.000+00$ \\
\hline $2-$ & 6 & E1 & $2.545-01$ & $2.280-01$ & $2.077-01$ & $1.913-01$ & $1.776-01$ & $1.659-01$ \\
\hline $2-$ & 7 & E1 & $1.535-01$ & $1.377-01$ & $1.257-01$ & $1.161-01$ & $1.080-01$ & $1.012-01$ \\
\hline $2-$ & 8 & E1 & $2.052-01$ & $1.844-01$ & $1.685-01$ & $1.557-01$ & $1.453-01$ & $1.365-01$ \\
\hline $2-$ & 9 & E1f & $1.236-05$ & $2.049-05$ & $3.419-05$ & $5.550-05$ & $8.662-05$ & $1.310-04$ \\
\hline $2-$ & 10 & M1 & $0.000+00$ & $0.000+00$ & $0.000+00$ & $0.000+00$ & $0.000+00$ & $0.000+00$ \\
\hline $3-$ & 4 & E2 & $0.000+00$ & $0.000+00$ & $0.000+00$ & $0.000+00$ & $0.000+00$ & $0.000+00$ \\
\hline $3-$ & 6 & M2 & $0.000+00$ & $0.000+00$ & $0.000+00$ & $0.000+00$ & $0.000+00$ & $0.000+00$ \\
\hline $3-$ & 7 & E1 & $2.040-01$ & $1.829-01$ & $1.668-01$ & $1.537-01$ & $1.428-01$ & $1.335-01$ \\
\hline $3-$ & 9 & E1f & $1.762-05$ & $3.985-05$ & 7.189-05 & $1.191-04$ & $1.876-04$ & 2.845-04 \\
\hline $4-$ & 5 & E2 & $0.000+00$ & $0.000+00$ & $0.000+00$ & $0.000+00$ & $0.000+00$ & $0.000+00$ \\
\hline $4-$ & 6 & E1f & $3.219-04$ & $5.590-04$ & $9.228-04$ & $1.439-03$ & $2.157-03$ & $3.109-03$ \\
\hline $4-$ & 7 & E1f & $1.616-05$ & $2.539-05$ & $3.808-05$ & 5.374-05 & $7.205-05$ & $9.140-05$ \\
\hline $4-$ & 8 & M2 & $0.000+00$ & $0.000+00$ & $0.000+00$ & $0.000+00$ & $0.000+00$ & $0.000+00$ \\
\hline $4-$ & 9 & E1 & $1.349+00$ & $1.207+00$ & $1.097+00$ & $1.008+00$ & $9.326-01$ & 8.685-01 \\
\hline $4-$ & 10 & E2 & $0.000+00$ & $0.000+00$ & $0.000+00$ & $0.000+00$ & $0.000+00$ & $0.000+00$ \\
\hline $5-$ & 6 & M2 & $0.000+00$ & $0.000+00$ & $0.000+00$ & $0.000+00$ & $0.000+00$ & $0.000+00$ \\
\hline $5-$ & 7 & E1f & $5.413-05$ & $8.827-05$ & $1.420-04$ & $2.205-04$ & $3.352-04$ & 4.974-04 \\
\hline $5-$ & 9 & E1 & $8.334-02$ & $8.690-02$ & $8.522-02$ & 8.206-02 & $7.857-02$ & $7.509-02$ \\
\hline $6-$ & 7 & M1 & $0.000+00$ & $0.000+00$ & $0.000+00$ & $0.000+00$ & $0.000+00$ & $0.000+00$ \\
\hline $6-$ & 8 & E2 & $0.000+00$ & $0.000+00$ & $0.000+00$ & $0.000+00$ & $0.000+00$ & $0.000+00$ \\
\hline $6-$ & 9 & M1 & $0.000+00$ & $0.000+00$ & $0.000+00$ & $0.000+00$ & $0.000+00$ & $0.000+00$ \\
\hline $6-$ & 10 & M2 & $0.000+00$ & $0.000+00$ & $0.000+00$ & $0.000+00$ & $0.000+00$ & $0.000+00$ \\
\hline $7-$ & 8 & M1 & $0.000+00$ & $0.000+00$ & $0.000+00$ & $0.000+00$ & $0.000+00$ & $0.000+00$ \\
\hline $7-$ & 9 & M1 & $0.000+00$ & $0.000+00$ & $0.000+00$ & $0.000+00$ & $0.000+00$ & $0.000+00$ \\
\hline 7 - & 10 & E1f & $3.949-05$ & $7.887-05$ & $1.359-04$ & $2.240-04$ & $3.543-04$ & 5.409-04 \\
\hline $8-$ & 9 & M1 & $0.000+00$ & $0.000+00$ & $0.000+00$ & $0.000+00$ & $0.000+00$ & $0.000+00$ \\
\hline $9-$ & 10 & E1 & $6.004-01$ & 5.540-01 & 5.186-01 & 4.870-01 & 4.590-01 & $4.340-01$ \\
\hline
\end{tabular}

configurations in the atomic model decreases the accuracy of the radiative rates. Excellent agreement, within $10 \%$, is found between the present calculation and the results from
Tachiev \& Froese Fischer (2002); the older calculations by Fawcett (1986) are higher than the latter two datasets by 10-15\%. Zhang \& Sampson (2002) A values are always 
Table 5. $A$ values for the O-like sequence (ClX to V XVI). E1: electric dipole transitions; E1f: spin-forbidden E1 transitions; E2: electric quadrupole transitions; M1: magnetic dipole transitions; M2: magnetic quadrupole transitions.

\begin{tabular}{|c|c|c|c|c|c|c|c|c|c|}
\hline Down & Up & Type & $\mathrm{ClX}$ & Ar XI & K XII & Ca XIII & Sc XIV & Ti XV & V XVI \\
\hline 1 - & 2 & M1 & $2.838+01$ & $6.688+01$ & $1.497+02$ & $3.198+02$ & $6.568 \mathrm{e}+02$ & $1.300+03$ & $2.486 \mathrm{e}+03$ \\
\hline 1 - & 3 & E2 & $7.912-04$ & $2.140-03$ & $5.437-03$ & $1.269-02$ & $2.746 \mathrm{e}-02$ & $5.557-02$ & $1.053 \mathrm{e}-01$ \\
\hline $1-$ & 4 & M1 & $1.162+02$ & $2.234+02$ & $4.162+02$ & $7.518+02$ & $1.322 \mathrm{e}+03$ & $2.271+03$ & $3.825 \mathrm{e}+03$ \\
\hline 1 - & 5 & E2 & 4.443-01 & 6.869-01 & $1.032+00$ & $1.493+00$ & $2.085 \mathrm{e}+00$ & $2.831+00$ & $3.744 \mathrm{e}+00$ \\
\hline 1 - & 6 & E1 & $1.499+10$ & $1.674+10$ & $1.858+10$ & $2.050+10$ & $2.253 e+10$ & $2.468+10$ & $2.696 \mathrm{e}+10$ \\
\hline $1-$ & 7 & E1 & $8.967+09$ & $1.020+10$ & $1.158+10$ & $1.311+10$ & $1.484 \mathrm{e}+10$ & $1.683+10$ & $1.911 \mathrm{e}+10$ \\
\hline $1-$ & 8 & M2 & $1.944+01$ & $2.452+01$ & $3.029+01$ & $3.685+01$ & $4.406 \mathrm{e}+01$ & $5.212+01$ & $6.104 \mathrm{e}+01$ \\
\hline $1-$ & 9 & E1f & $6.602+08$ & $1.029+09$ & $1.543+09$ & $2.232+09$ & $3.121 \mathrm{e}+09$ & $4.229+09$ & $5.566 \mathrm{e}+09$ \\
\hline $1-$ & 10 & E2 & $5.030+03$ & $8.749+03$ & $1.113+04$ & $1.583+04$ & $2.189 \mathrm{e}+04$ & $2.953+04$ & $3.894 \mathrm{e}+04$ \\
\hline $2-$ & 3 & M1 & $1.879+00$ & $2.942+00$ & $4.103+00$ & $4.489+00$ & $3.443 e+00$ & $1.386+00$ & $4.913 \mathrm{e}-02$ \\
\hline $2-$ & 4 & M1 & $2.261+01$ & $3.852+01$ & $6.267+01$ & $9.730+01$ & $1.445 \mathrm{e}+02$ & $2.067+02$ & $2.855 \mathrm{e}+02$ \\
\hline $2-$ & 5 & M1 & $1.370+03$ & $2.575+03$ & $4.675+03$ & $8.197+03$ & $1.393 e+04$ & $2.306+04$ & $3.728 \mathrm{e}+04$ \\
\hline $2-$ & 6 & E1 & $4.708+09$ & $5.197+09$ & $5.697+09$ & $6.202+09$ & $6.715 e+09$ & $7.234+09$ & $7.760 \mathrm{e}+09$ \\
\hline $2-$ & 7 & E1 & $4.995+09$ & $5.581+09$ & $6.194+09$ & $6.837+09$ & $7.512 \mathrm{e}+09$ & $8.217+09$ & $8.954 \mathrm{e}+09$ \\
\hline $2-$ & 8 & E1 & $2.070+10$ & $2.332+10$ & $2.613+10$ & $2.917+10$ & $3.247 \mathrm{e}+10$ & $3.607+10$ & $4.002 \mathrm{e}+10$ \\
\hline $2-$ & 9 & E1f & $1.869+07$ & $3.151+07$ & $5.134+07$ & $8.138+07$ & $1.259 \mathrm{e}+08$ & $1.908+08$ & $2.836 \mathrm{e}+08$ \\
\hline $2-$ & 10 & M1 & $3.061+02$ & $6.092+02$ & $9.874+02$ & $1.681+03$ & $2.776 e+03$ & $4.460+03$ & $6.990 \mathrm{e}+03$ \\
\hline $3-$ & 4 & E2 & $1.300-03$ & $1.990-03$ & $2.996-03$ & $4.473-03$ & $6.683 e-03$ & $1.011-02$ & $1.567 \mathrm{e}-02$ \\
\hline $3-$ & 6 & M2 & $5.601+00$ & $7.640+00$ & $1.030+01$ & $1.377+01$ & $1.827 \mathrm{e}+01$ & $2.408+01$ & $3.155 \mathrm{e}+01$ \\
\hline $3-$ & 7 & E1 & $6.476+09$ & $7.202+09$ & $7.950+09$ & $8.729+09$ & $9.547 \mathrm{e}+09$ & $1.040+10$ & $1.131 \mathrm{e}+10$ \\
\hline $3-$ & 9 & E1f & $4.039+07$ & $6.772+07$ & $1.097+08$ & $1.721+08$ & $2.613 e+08$ & $3.844+08$ & $5.470 \mathrm{e}+08$ \\
\hline $4-$ & 5 & E2 & $8.548+00$ & $9.694+00$ & $1.110+01$ & $1.281+01$ & $1.496 \mathrm{e}+01$ & $1.790+01$ & $2.198 \mathrm{e}+01$ \\
\hline $4-$ & 6 & E1f & $1.030+08$ & $1.630+08$ & $2.481+08$ & $3.639+08$ & $5.159 \mathrm{e}+08$ & $7.088+08$ & $9.460 \mathrm{e}+08$ \\
\hline $4-$ & 7 & E1f & $4.529+06$ & $5.998+06$ & $7.279+06$ & $7.916+06$ & $7.419 \mathrm{e}+06$ & $5.488+06$ & $2.476 \mathrm{e}+06$ \\
\hline 4 - & 8 & M2 & $2.788+01$ & $3.846+01$ & $5.226+01$ & $7.023+01$ & $9.358 \mathrm{e}+01$ & $1.236+02$ & $1.622 \mathrm{e}+02$ \\
\hline $4-$ & 9 & E1 & $6.671+10$ & $7.359+10$ & $8.065+10$ & $8.787+10$ & $9.529 \mathrm{e}+10$ & $1.029+11$ & $1.108 \mathrm{e}+11$ \\
\hline 4 - & 10 & E2 & $3.653+05$ & $4.426+05$ & $4.083+05$ & $4.299+05$ & $4.517 \mathrm{e}+05$ & $4.737+05$ & $4.962 \mathrm{e}+05$ \\
\hline $5-$ & 6 & M2 & $2.374+00$ & $2.928+00$ & $3.503+00$ & $4.073+00$ & $4.604 \mathrm{e}+00$ & $5.047+00$ & $5.363 \mathrm{e}+00$ \\
\hline $5-$ & 7 & E1f & $2.097+07$ & $3.521+07$ & $5.729+07$ & $9.019+07$ & $1.374 \mathrm{e}+08$ & $2.019+08$ & $2.858 \mathrm{e}+08$ \\
\hline $5-$ & 9 & E1 & $4.606+09$ & $5.165+09$ & $5.735+09$ & $6.314+09$ & $6.901 e+09$ & $7.488+09$ & $8.072 \mathrm{e}+09$ \\
\hline $6-$ & 7 & M1 & $1.854+01$ & $4.204+01$ & $8.847+01$ & $1.782+02$ & $3.442 \mathrm{e}+02$ & $6.361+02$ & $1.131 \mathrm{e}+03$ \\
\hline $6-$ & 8 & E2 & 8.904-04 & $2.647-03$ & $7.195-03$ & $1.857-02$ & $4.588 \mathrm{e}-02$ & $1.077-01$ & $2.422 \mathrm{e}-01$ \\
\hline $6-$ & 9 & M1 & $2.045+02$ & $3.947+02$ & $7.340+02$ & $1.322+03$ & $2.318 \mathrm{e}+03$ & $3.970+03$ & $6.662 \mathrm{e}+03$ \\
\hline $6-$ & 10 & M2 & $4.574+02$ & $7.124+02$ & $7.731+02$ & $9.862+02$ & $1.246 \mathrm{e}+03$ & $1.561+03$ & $1.945 \mathrm{e}+03$ \\
\hline 7 - & 8 & M1 & $7.921+00$ & $1.870+01$ & $4.183+01$ & $8.973+01$ & $1.873 \mathrm{e}+02$ & $3.771+02$ & $7.392 \mathrm{e}+02$ \\
\hline 7 - & 9 & M1 & $1.056+02$ & $1.965+02$ & $3.512+02$ & $6.053+02$ & $1.010 \mathrm{e}+03$ & $1.641+03$ & $2.600 \mathrm{e}+03$ \\
\hline $7-$ & 10 & E1f & $2.190+08$ & $4.119+08$ & $6.085+08$ & $9.706+08$ & $1.509 \mathrm{e}+09$ & $2.296+09$ & $3.424 \mathrm{e}+09$ \\
\hline $8-$ & 9 & M1 & $1.277+02$ & $2.327+02$ & $4.058+02$ & $6.790+02$ & $1.094 \mathrm{e}+03$ & $1.704+03$ & $2.574 \mathrm{e}+03$ \\
\hline 9 - & 10 & E1 & $5.905+10$ & $7.291+10$ & $7.454+10$ & $8.284+10$ & $9.152 \mathrm{e}+10$ & $1.006+11$ & $1.100 \mathrm{e}+11$ \\
\hline
\end{tabular}

overestimated by $15 \%$ to $40 \%$, the differences being largest for the forbidden transitions. Similar overestimations are found for the Cheng et al. (1979) data.
In general, agreement between the present calculation and results from the most sophisticated calculations available in the literature is within $10 \%$. 
Table 6. Weighted oscillator strengths for the O-like sequence (Cl X to V XVI). E1: electric dipole transitions; E1f: spin-forbidden E1 transitions; E2: electric quadrupole transitions; M1: magnetic dipole transitions; M2: magnetic quadrupole transitions.

\begin{tabular}{|c|c|c|c|c|c|c|c|c|c|}
\hline Down & Up & Type & $\mathrm{Clx}$ & Ar XI & K XII & Ca XIII & Sc XIV & Ti XV & V XVI \\
\hline $1-$ & 2 & M1 & $0.000+00$ & $0.000+00$ & $0.000+00$ & $0.000+00$ & $0.000+00$ & $0.000+00$ & $0.000+00$ \\
\hline $1-$ & 3 & E2 & $0.000+00$ & $0.000+00$ & $0.000+00$ & $0.000+00$ & $0.000+00$ & $0.000+00$ & $0.000+00$ \\
\hline $1-$ & 4 & M1 & $0.000+00$ & $0.000+00$ & $0.000+00$ & $0.000+00$ & $0.000+00$ & $0.000+00$ & $0.000+00$ \\
\hline $1-$ & 5 & E2 & $0.000+00$ & $0.000+00$ & $0.000+00$ & $0.000+00$ & $0.000+00$ & $0.000+00$ & $0.000+00$ \\
\hline $1-$ & 6 & E1 & 4.743-01 & 4.476-01 & $4.238-01$ & $4.022-01$ & $3.827-01$ & 3.649-01 & $3.485-01$ \\
\hline $1-$ & 7 & E1 & $1.638-01$ & $1.563-01$ & $1.501-01$ & $1.448-01$ & $1.406-01$ & $1.372-01$ & $1.348-01$ \\
\hline $1-$ & 8 & M2 & $0.000+00$ & $0.000+00$ & $0.000+00$ & $0.000+00$ & $0.000+00$ & $0.000+00$ & $0.000+00$ \\
\hline $1-$ & 9 & E1f & $6.566-03$ & $8.677-03$ & $1.114-02$ & $1.389-02$ & $1.685-02$ & $1.990-02$ & $2.291-02$ \\
\hline $1-$ & 10 & E2 & $0.000+00$ & $0.000+00$ & $0.000+00$ & $0.000+00$ & $0.000+00$ & $0.000+00$ & $0.000+00$ \\
\hline $2-$ & 3 & M1 & $0.000+00$ & $0.000+00$ & $0.000+00$ & $0.000+00$ & $0.000+00$ & $0.000+00$ & $0.000+00$ \\
\hline $2-$ & 4 & M1 & $0.000+00$ & $0.000+00$ & $0.000+00$ & $0.000+00$ & $0.000+00$ & $0.000+00$ & $0.000+00$ \\
\hline $2-$ & 5 & M1 & $0.000+00$ & $0.000+00$ & $0.000+00$ & $0.000+00$ & $0.000+00$ & $0.000+00$ & $0.000+00$ \\
\hline $2-$ & 6 & E1 & $1.558-01$ & $1.469-01$ & $1.390-01$ & $1.319-01$ & $1.256-01$ & $1.198-01$ & $1.145-01$ \\
\hline $2-$ & 7 & E1 & $9.538-02$ & $9.025-02$ & $8.572-02$ & $8.167-02$ & $7.802-02$ & $7.470-02$ & $7.165-02$ \\
\hline $2-$ & 8 & E1 & $1.289-01$ & $1.224-01$ & $1.167-01$ & $1.118-01$ & $1.074-01$ & $1.036-01$ & $1.002-01$ \\
\hline $2-$ & 9 & E1f & $1.920-04$ & $2.767-04$ & $3.891-04$ & $5.370-04$ & 7.288-04 & $9.745-04$ & $1.285-03$ \\
\hline $2-$ & 10 & M1 & $0.000+00$ & $0.000+00$ & $0.000+00$ & $0.000+00$ & $0.000+00$ & $0.000+00$ & $0.000+00$ \\
\hline $3-$ & 4 & E2 & $0.000+00$ & $0.000+00$ & $0.000+00$ & $0.000+00$ & $0.000+00$ & $0.000+00$ & $0.000+00$ \\
\hline $3-$ & 6 & M2 & $0.000+00$ & $0.000+00$ & $0.000+00$ & $0.000+00$ & $0.000+00$ & $0.000+00$ & $0.000+00$ \\
\hline $3-$ & 7 & E1 & $1.254-01$ & $1.182-01$ & $1.117-01$ & $1.058-01$ & $1.004-01$ & $9.534-02$ & $9.074-02$ \\
\hline $3-$ & 9 & E1f & 4.192-04 & $6.010-04$ & $8.405-04$ & $1.147-03$ & $1.526-03$ & $1.976-03$ & $2.483-03$ \\
\hline $4-$ & 5 & E2 & $0.000+00$ & $0.000+00$ & $0.000+00$ & $0.000+00$ & $0.000+00$ & $0.000+00$ & $0.000+00$ \\
\hline $4-$ & 6 & E1f & $4.335-03$ & $5.832-03$ & $7.631-03$ & $9.714-03$ & $1.205-02$ & $1.459-02$ & $1.728-02$ \\
\hline $4-$ & 7 & E1f & $1.095-04$ & $1.221-04$ & $1.261-04$ & $1.177-04$ & $9.541-05$ & $6.142-05$ & $2.426-05$ \\
\hline $4-$ & 8 & M2 & $0.000+00$ & $0.000+00$ & $0.000+00$ & $0.000+00$ & $0.000+00$ & $0.000+00$ & $0.000+00$ \\
\hline $4-$ & 9 & E1 & $8.127-01$ & $7.637-01$ & $7.201-01$ & $6.808-01$ & $6.453-01$ & $6.126-01$ & 5.831-01 \\
\hline $4-$ & 10 & E2 & $0.000+00$ & $0.000+00$ & $0.000+00$ & $0.000+00$ & $0.000+00$ & $0.000+00$ & $0.000+00$ \\
\hline $5-$ & 6 & M2 & $0.000+00$ & $0.000+00$ & $0.000+00$ & $0.000+00$ & $0.000+00$ & $0.000+00$ & $0.000+00$ \\
\hline $5-$ & 7 & E1f & $7.239-04$ & $1.023-03$ & $1.419-03$ & $1.921-03$ & $2.539-03$ & $3.267-03$ & $4.084-03$ \\
\hline $5-$ & 9 & E1 & $7.176-02$ & $6.865-02$ & $6.572-02$ & $6.298-02$ & $6.040-02$ & $5.795-02$ & $5.565-02$ \\
\hline $6-$ & 7 & M1 & $0.000+00$ & $0.000+00$ & $0.000+00$ & $0.000+00$ & $0.000+00$ & $0.000+00$ & $0.000+00$ \\
\hline $6-$ & 8 & E2 & $0.000+00$ & $0.000+00$ & $0.000+00$ & $0.000+00$ & $0.000+00$ & $0.000+00$ & $0.000+00$ \\
\hline $6-$ & 9 & M1 & $0.000+00$ & $0.000+00$ & $0.000+00$ & $0.000+00$ & $0.000+00$ & $0.000+00$ & $0.000+00$ \\
\hline $6-$ & 10 & M2 & $0.000+00$ & $0.000+00$ & $0.000+00$ & $0.000+00$ & $0.000+00$ & $0.000+00$ & $0.000+00$ \\
\hline $7-$ & 8 & M1 & $0.000+00$ & $0.000+00$ & $0.000+00$ & $0.000+00$ & $0.000+00$ & $0.000+00$ & $0.000+00$ \\
\hline $7-$ & 9 & M1 & $0.000+00$ & $0.000+00$ & $0.000+00$ & $0.000+00$ & $0.000+00$ & $0.000+00$ & $0.000+00$ \\
\hline $7-$ & 10 & E1f & $8.015-04$ & $1.198-03$ & $1.634-03$ & $2.263-03$ & $3.080-03$ & $4.127-03$ & $5.452-03$ \\
\hline $8-$ & 9 & M1 & $0.000+00$ & $0.000+00$ & $0.000+00$ & $0.000+00$ & $0.000+00$ & $0.000+00$ & $0.000+00$ \\
\hline 9 - & 10 & E1 & 4.117-01 & 4.036-01 & $3.734-01$ & $3.569-01$ & $3.419-01$ & $3.281-01$ & $3.149-01$ \\
\hline
\end{tabular}

Data calculated for Ca XIII in the present work are in excellent agreement (within 10\%) with results from Tachiev \& Froese Fischer (2002) and Vilkas et al. (1994); Fawcett (1986) is slightly higher than the present calculation while Zhang \& Sampson (2002) overestimate $A$ values by up to a factor of 2 in the worst cases. Cheng et al. (1979) results are also higher and 
Table 7. A values for the O-like sequence (Cr XVII to Zn XXIII). E1: electric dipole transitions; E1f: spin-forbidden E1 transitions; E2: electric quadrupole transitions; M1: magnetic dipole transitions; M2: magnetic quadrupole transitions.

\begin{tabular}{|c|c|c|c|c|c|c|c|c|c|}
\hline Down & Up & ype & Cr XVII & Mn XVIII & Fe XIX & Co XX & Ni XXI & $\mathrm{Cu}$ XXII & Zn XXIII \\
\hline $1-$ & 2 & E2 & $872-01$ & $3.134-01$ & 4.976-01 & 7.399-01 & $1.066+00$ & $1.469+00$ & $1.943+00$ \\
\hline $1-$ & 3 & M1 & $609+03$ & $.306+03$ & $1.458+04$ & $2.488+04$ & $4.177+04$ & $6.850+04$ & $1.103+05$ \\
\hline $1-$ & 4 & M1 & $.327+03$ & $1.030+04$ & $1.652+04$ & $2.617+04$ & $4.083+04$ & $6.302+04$ & $9.588+04$ \\
\hline 1 - & 5 & E2 & $.833+00$ & $6.110+00$ & $7.581+00$ & $9.261+00$ & $1.116+01$ & $1.332+01$ & $1.565+01$ \\
\hline 1 - & 6 & E1 & $2.941+10$ & $3.205+10$ & $3.492+10$ & $3.805+10$ & $4.149+10$ & $4.532+10$ & $4.955+10$ \\
\hline 1 - & 7 & E1 & $177+10$ & $2.488+10$ & $2.855+10$ & $3.287+10$ & $3.801+10$ & $4.408+10$ & $5.125+10$ \\
\hline 1 - & 8 & M2 & $7.092+01$ & $8.189+01$ & $9.418+01$ & $1.079+02$ & $1.241+02$ & $1.415+02$ & $1.622+02$ \\
\hline $1-$ & 9 & E1f & $7.126+09$ & $8.890+09$ & $1.083+10$ & $1.289+10$ & $1.503+10$ & $1.717+10$ & $1.927+10$ \\
\hline 1 - & 10 & E2 & $5.029+04$ & $6.374+04$ & $7.949+04$ & $9.766+04$ & $1.185+05$ & $1.421+05$ & $1.689+05$ \\
\hline $2-$ & 3 & M1 & $1.962-01$ & $5.835+00$ & $4.117+01$ & $1.793+02$ & $5.852+02$ & $1.597+03$ & $3.863+03$ \\
\hline $2-$ & 4 & E2 & $2.514-02$ & 4.196-02 & $7.269-02$ & $1.324-01$ & $2.435-01$ & $4.596-01$ & $8.726-01$ \\
\hline $2-$ & 6 & M2 & $4.107+01$ & $5.314+01$ & $6.836+01$ & $8.755+01$ & $1.110+02$ & $1.416+02$ & $1.793+02$ \\
\hline $2-$ & 7 & E1 & $1.228+10$ & $1.334+10$ & $1.451+10$ & $1.584+10$ & $1.736+10$ & $1.911+10$ & $2.116+10$ \\
\hline $2-$ & 9 & E1f & $7.523+08$ & $9.989+08$ & $1.281+09$ & $1.589+09$ & $1.907+09$ & $2.218+09$ & $2.509+09$ \\
\hline $3-$ & 4 & M1 & $3.823+02$ & $4.976+02$ & $6.320+02$ & $7.899+02$ & $9.573+02$ & $1.147+03$ & $1.339+03$ \\
\hline $3-$ & 5 & M1 & $5.904+04$ & $9.180+04$ & $1.404+05$ & $2.119+05$ & $3.151+05$ & $4.640+05$ & $6.731+05$ \\
\hline $3-$ & 6 & E1 & $8.293+09$ & $8.834+09$ & $9.383+09$ & $9.940+09$ & $1.050+10$ & $1.107+10$ & $1.165+10$ \\
\hline $3-$ & 7 & E1 & $9.725+09$ & $1.051+10$ & $1.133+10$ & $1.215+10$ & $1.299+10$ & $1.381+10$ & $1.462+10$ \\
\hline $3-$ & 8 & E1 & $4.434+10$ & $4.913+10$ & $5.445+10$ & $6.033+10$ & $6.692+10$ & $7.428+10$ & $8.253+10$ \\
\hline $3-$ & 9 & E1f & $4.143+08$ & $5.956+08$ & $8.436+08$ & $1.178+09$ & $1.623+09$ & $2.204+09$ & $2.954+09$ \\
\hline $3-$ & 10 & M1 & $1.071+04$ & $1.610+04$ & $2.374+04$ & $3.443+04$ & $4.917+04$ & $6.916+04$ & $9.608+04$ \\
\hline $4-$ & 5 & $\mathrm{E} 2$ & $2.780+01$ & $3.629+01$ & $4.889+01$ & $6.776+01$ & $9.684+01$ & $1.413+02$ & $2.086+02$ \\
\hline $4-$ & 6 & E1f & $229+09$ & 1.55 & $1.935+09$ & $2.352+09$ & $8+09$ & +09 & $4+09$ \\
\hline $4-$ & 7 & E1f & $950+04$ & $2.387+06$ & $5+07$ & $5.564+07$ & $1.367+08$ & +08 & $5.269+08$ \\
\hline $4-$ & 8 & M2 & $16+02$ & 2.746 & & +02 & $5.863+02$ & $7.499+02$ & $9.592+02$ \\
\hline $4-$ & 9 & E1 & $190+11$ & $1.274+11$ & $63+11$ & $1.457+11$ & $1.559+11$ & $1.664+11$ & $1.779+11$ \\
\hline $4-$ & 10 & E2 & $194+05$ & $5.431+05$ & $5.681+05$ & $5.942+05$ & $6.226+05$ & $6.519+05$ & $6.847+05$ \\
\hline $5-$ & 6 & M2 & $20+00$ & $5.506+00$ & $28+00$ & $5.003+00$ & $4.592+00$ & $4.074+00$ & $5+00$ \\
\hline $5-$ & 7 & E1f & $3.889+08$ & $5.079+08$ & $6.364+08$ & $7.642+08$ & $8.820+08$ & $9.787+08$ & $1.049+09$ \\
\hline $5-$ & 9 & E1 & $8.651+09$ & $9.224+09$ & $9.798+09$ & $1.038+10$ & $1.098+10$ & $1.160+10$ & $1.227+10$ \\
\hline $6-$ & 7 & M1 & $1.939+03$ & $3.213+03$ & $5.154+03$ & $7.965+03$ & $1.206+04$ & $1.767+04$ & $2.521+04$ \\
\hline $6-$ & 8 & E2 & $5.246-01$ & $1.097+00$ & $2.225+00$ & $4.351+00$ & $8.383+00$ & $1.569+01$ & $2.877+01$ \\
\hline $6-$ & 9 & M1 & $1.098+04$ & $1.781+04$ & $2.850+04$ & $4.502+04$ & $7.036+04$ & $1.085+05$ & $1.657+05$ \\
\hline $6-$ & 10 & M2 & $2.411+03$ & $2.976+03$ & $3.663+03$ & $4.498+03$ & $5.520+03$ & $6.750+03$ & $8.269+03$ \\
\hline 7 - & 8 & M1 & $1.414+03$ & $2.645+03$ & $4.853+03$ & $8.723+03$ & $1.546+04$ & $2.692+04$ & $4.612+04$ \\
\hline 7 - & 9 & M1 & $4.030+03$ & $6.131+03$ & $9.173+03$ & $1.355+04$ & $1.975+04$ & $2.840+04$ & $4.058+04$ \\
\hline 7 - & 10 & E1f & $5.016+09$ & $7.231+09$ & $1.026+10$ & $1.437+10$ & $1.983+10$ & $2.695+10$ & $3.618+10$ \\
\hline $8-$ & 9 & M1 & $3.773+03$ & $5.379+03$ & $7.462+03$ & $1.012+04$ & $1.335+04$ & $1.714+04$ & $2.154+04$ \\
\hline $9-$ & 10 & E1 & $1.198+11$ & $1.299+11$ & $1.403+11$ & $1.509+11$ & $1.615+11$ & $1.720+11$ & $1.823+11$ \\
\hline
\end{tabular}

are closer to those from Zhang \& Samspson (2002). Similar results are also found for Si VII: although Fawcett (1986), Cheng et al. (1979) and Zhang \& Sampson (2002) are always higher than the present calculations, results from Tachiev \& Froese Fischer (2002) and Vilkas et al. (1994) usually agree within $10 \%$ with the present results, although a very few transitions show differences up to $25 \%$.
Fe XIX has been paid a greater consideration in the literature due to the strength and importance of its spectral lines. Table 11 reports the comparison between the present calculation and other five datasets. Butler \& Zeippen (2001) data show excellent agreement (within 10\%) with the present calculation for all transitions; those from Vilkas et al. (1994) also are in excellent agreement, except for a few, weak 
Table 8. Weighted oscillator strengths for the O-like sequence (Cr XVII to Zn XXIII). E1: electric dipole transitions; E1f: spin-forbidden E1 transitions; E2: electric quadrupole transitions; M1: magnetic dipole transitions; M2: magnetic quadrupole transitions.

\begin{tabular}{|c|c|c|c|c|c|c|c|c|c|}
\hline Down & Up & Type & Cr XVII & Mn XVIII & Fe XIX & Co XX & Ni XXI & $\mathrm{Cu}$ XXII & Zn XXIII \\
\hline $1-$ & 2 & E2 & $0.000+00$ & $0.000+00$ & $0.000+00$ & $0.000+00$ & $0.000+00$ & $0.000+00$ & $0.000+00$ \\
\hline $1-$ & 3 & M1 & $0.000+00$ & $0.000+00$ & $0.000+00$ & $0.000+00$ & $0.000+00$ & $0.000+00$ & $0.000+00$ \\
\hline $1-$ & 4 & M1 & $0.000+00$ & $0.000+00$ & $0.000+00$ & $0.000+00$ & $0.000+00$ & $0.000+00$ & $0.000+00$ \\
\hline $1-$ & 5 & E2 & $0.000+00$ & $0.000+00$ & $0.000+00$ & $0.000+00$ & $0.000+00$ & $0.000+00$ & $0.000+00$ \\
\hline $1-$ & 6 & E1 & $3.336-01$ & 3.199-01 & $3.075-01$ & $2.962-01$ & $2.860-01$ & $2.769-01$ & $2.687-01$ \\
\hline $1-$ & 7 & E1 & $1.332-01$ & $1.324-01$ & $1.325-01$ & $1.333-01$ & $1.349-01$ & $1.372-01$ & $1.401-01$ \\
\hline $1-$ & 8 & M2 & $0.000+00$ & $0.000+00$ & $0.000+00$ & $0.000+00$ & $0.000+00$ & $0.000+00$ & $0.000+00$ \\
\hline $1-$ & 9 & E1f & $2.574-02$ & $2.825-02$ & $3.033-02$ & $3.185-02$ & $3.278-02$ & $3.309-02$ & $3.279-02$ \\
\hline $1-$ & 10 & E2 & $0.000+00$ & $0.000+00$ & $0.000+00$ & $0.000+00$ & $0.000+00$ & $0.000+00$ & $0.000+00$ \\
\hline $2-$ & 3 & M1 & $0.000+00$ & $0.000+00$ & $0.000+00$ & $0.000+00$ & $0.000+00$ & $0.000+00$ & $0.000+00$ \\
\hline $2-$ & 4 & $\mathrm{E} 2$ & $0.000+00$ & $0.000+00$ & $0.000+00$ & $0.000+00$ & $0.000+00$ & $0.000+00$ & $0.000+00$ \\
\hline $2-$ & 6 & M2 & $0.000+00$ & $0.000+00$ & $0.000+00$ & $0.000+00$ & $0.000+00$ & $0.000+00$ & $0.000+00$ \\
\hline $2-$ & 7 & E1 & 8.646-02 & $8.250-02$ & 7.894-02 & 7.586-02 & 7.319-02 & 7.097-02 & $6.925-02$ \\
\hline $2-$ & 9 & E1f & $3.024-03$ & $3.562-03$ & $4.055-03$ & $4.464-03$ & $4.754-03$ & $4.904-03$ & 4.914-03 \\
\hline $3-$ & 4 & M1 & $0.000+00$ & $0.000+00$ & $0.000+00$ & $0.000+00$ & $0.000+00$ & $0.000+00$ & $0.000+00$ \\
\hline $3-$ & 5 & M1 & $0.000+00$ & $0.000+00$ & $0.000+00$ & $0.000+00$ & $0.000+00$ & $0.000+00$ & $0.000+00$ \\
\hline $3-$ & 6 & E1 & $1.097-01$ & $1.054-01$ & 1.013-01 & $9.757-02$ & $9.409-02$ & $9.090-02$ & $8.786-02$ \\
\hline $3-$ & 7 & E1 & $6.885-02$ & $6.613-02$ & $6.361-02$ & $6.112-02$ & $5.873-02$ & $5.633-02$ & $5.399-02$ \\
\hline $3-$ & 8 & E1 & $9.722-02$ & $9.460-02$ & $9.232-02$ & $9.030-02$ & $8.856-02$ & 8.704-02 & $8.575-02$ \\
\hline $3-$ & 9 & E1f & $1.673-03$ & $2.152-03$ & $2.735-03$ & $3.436-03$ & $4.269-03$ & $5.237-03$ & $6.348-03$ \\
\hline $3-$ & 10 & M1 & $0.000+00$ & $0.000+00$ & $0.000+00$ & $0.000+00$ & $0.000+00$ & $0.000+00$ & $0.000+00$ \\
\hline $4-$ & 5 & E2 & $0.000+00$ & $0.000+00$ & $0.000+00$ & $0.000+00$ & $0.000+00$ & $0.000+00$ & $0.000+00$ \\
\hline $4-$ & 6 & E1f & $2.005-02$ & $2.281-02$ & $2.552-02$ & $2.810-02$ & $3.051-02$ & $3.272-02$ & $3.471-02$ \\
\hline $4-$ & 7 & E1f & 8.574-07 & $1.818-05$ & $1.139-04$ & $3.354-04$ & 7.368-04 & $1.374-03$ & $2.298-03$ \\
\hline $4-$ & 8 & M2 & $0.000+00$ & $0.000+00$ & $0.000+00$ & $0.000+00$ & $0.000+00$ & $0.000+00$ & $0.000+00$ \\
\hline $4-$ & 9 & E1 & $5.563-01$ & $5.312-01$ & $5.082-01$ & $4.872-01$ & 4.681-01 & 4.499-01 & 4.334-01 \\
\hline $4-$ & 10 & E2 & $0.000+00$ & $0.000+00$ & $0.000+00$ & $0.000+00$ & $0.000+00$ & $0.000+00$ & $0.000+00$ \\
\hline $5-$ & 6 & M2 & $0.000+00$ & $0.000+00$ & $0.000+00$ & $0.000+00$ & $0.000+00$ & $0.000+00$ & $0.000+00$ \\
\hline $5-$ & 7 & E1f & $4.948-03$ & $5.803-03$ & $6.582-03$ & $7.222-03$ & $7.677-03$ & $7.915-03$ & 7.941-03 \\
\hline $5-$ & 9 & E1 & 5.349-02 & $5.147-02$ & 4.964-02 & $4.801-02$ & $4.662-02$ & $4.542-02$ & 4.449-02 \\
\hline $6-$ & 7 & M1 & $0.000+00$ & $0.000+00$ & $0.000+00$ & $0.000+00$ & $0.000+00$ & $0.000+00$ & $0.000+00$ \\
\hline $6-$ & 8 & E2 & $0.000+00$ & $0.000+00$ & $0.000+00$ & $0.000+00$ & $0.000+00$ & $0.000+00$ & $0.000+00$ \\
\hline $6-$ & 9 & M1 & $0.000+00$ & $0.000+00$ & $0.000+00$ & $0.000+00$ & $0.000+00$ & $0.000+00$ & $0.000+00$ \\
\hline $6-$ & 10 & M2 & $0.000+00$ & $0.000+00$ & $0.000+00$ & $0.000+00$ & $0.000+00$ & $0.000+00$ & $0.000+00$ \\
\hline $7-$ & 8 & M1 & $0.000+00$ & $0.000+00$ & $0.000+00$ & $0.000+00$ & $0.000+00$ & $0.000+00$ & $0.000+00$ \\
\hline $7-$ & 9 & M1 & $0.000+00$ & $0.000+00$ & $0.000+00$ & $0.000+00$ & $0.000+00$ & $0.000+00$ & $0.000+00$ \\
\hline $7-$ & 10 & E1f & $7.110-03$ & $9.158-03$ & $1.165-02$ & $1.466-02$ & $1.822-02$ & $2.236-02$ & $2.712-02$ \\
\hline $8-$ & 9 & M1 & $0.000+00$ & $0.000+00$ & $0.000+00$ & $0.000+00$ & $0.000+00$ & $0.000+00$ & $0.000+00$ \\
\hline $9-$ & 10 & E1 & $3.028-01$ & 2.913-01 & $2.803-01$ & 2.696-01 & $2.591-01$ & $2.486-01$ & 2.382-01 \\
\hline
\end{tabular}

$A$ values. Data from Lolergue et al. (1985) tend to be higher than those of the latter calculations, but only by a limited amount. Discrepancies are also limited between the latter calculations and those from Fawcett (1986), Cheng et al. (1979), and Zhang \& Sampson (2002), although the latter seems provide slightly overestimated $A$ values for intercombination transitions.

The only experimental measurements of transition rates in the O-like sequence we are aware of are those by Flaig \& Schartner (1985), who measured the absorption oscillator strengths for the $2 s^{2} 2 p^{4}{ }^{1} D_{2}-2 s 2 p^{5}{ }^{1} P_{1}$ (4-9) and the
$2 s^{2} 2 p^{4}{ }^{1} S_{0}-2 s 2 p^{5}{ }^{1} P_{1}(5-9)$ transitions in Si VII. We have compared $A$ values derived from these measurements using observed wavelengths from Edlen (1983) with those in the present computation and in earlier works. Results are listed in Table 12. This comparison is even more important since it involves one of the ions for which the present energy levels were less accurate than those from earlier calculations. Results show that good agreement (within 10\%) is found between the measured $A$ value for the 4-9 transition and the results obtained in the present work, Tachiev \& Froese Fischer (2002) and Vilkas et al. (1994), while Zhang \& Sampson (2002) and 
Table 9. Comparison between different calculations of O-like radiative data: forbidden transitions within the Si VII ground configuration. 24C: present calculation; 6C: 6-configuration calculation described in Bhatia \& Landi (2003a); G97: Galavis et al. (1997); TFF02: Tachiev \& Froese Fischer (2002); BZ88: Baluja \& Zeippen (1988); FS83: Froese Fischer \& Saha (1983) (corrected with the experimental wavelengths); C79: Cheng et al. (1979).

\begin{tabular}{rrrrrrrr}
\hline \hline Transition & $24 \mathrm{C}$ & $6 \mathrm{C}$ & $\mathrm{G} 97$ & TFF02 & BZ88 & FS83 & C79 \\
\hline${ }^{3} \mathrm{P}_{2}-{ }^{3} \mathrm{P}_{1}$ & $1.467+00$ & $1.749+00$ & $1.465+00$ & $1.470+00$ & $1.472+00$ & $1.470+00$ & $1.430+00$ \\
${ }^{3} \mathrm{P}_{2}-{ }^{3} \mathrm{P}_{0}$ & $2.173-05$ & $2.762-05$ & $2.072-05$ & $2.007-05$ & $2.070-05$ & $2.152-05$ & $1.828-05$ \\
${ }^{3} \mathrm{P}_{1}-{ }^{3} \mathrm{P}_{0}$ & $1.944-01$ & $2.227-01$ & $1.964-01$ & $1.862-01$ & $1.973-01$ & $1.964-01$ & $1.670-01$ \\
${ }^{3} \mathrm{P}_{2}-{ }^{1} \mathrm{D}_{2}$ & $1.180+01$ & $1.469+01$ & $1.232+01$ & $1.257+01$ & $1.183+01$ & $1.173+01$ & $1.456+01$ \\
${ }^{3} \mathrm{P}_{1}-{ }^{1} \mathrm{D}_{2}$ & $3.028+00$ & $3.746+00$ & $3.139+00$ & $3.210+00$ & $3.016+00$ & $2.986+00$ & $3.910+00$ \\
${ }^{3} \mathrm{P}_{0}-{ }^{1} \mathrm{D}_{2}$ & $2.815-04$ & $4.822-04$ & $2.110-04$ & & $2.270-04$ & $2.273-04$ & $3.547-04$ \\
${ }^{3} \mathrm{P}_{2}-{ }^{1} \mathrm{~S}_{0}$ & $8.666-02$ & $7.006-02$ & $1.032-01$ & $1.025-01$ & $9.810-02$ & $9.003-02$ & $8.085-02$ \\
${ }^{3} \mathrm{P}_{1}-{ }^{1} \mathrm{~S}_{0}$ & $1.510+02$ & $1.557+02$ & $1.397+02$ & $1.423+02$ & $1.414+02$ & $1.396+02$ & $1.539+02$ \\
${ }^{1} \mathrm{D}_{2}-{ }^{1} \mathrm{~S}_{0}$ & $5.876+00$ & $3.220+00$ & $5.721+00$ & & $5.792+00$ & $5.894+00$ & $3.341+00$ \\
\hline
\end{tabular}

Table 10. Comparison between different calculations of O-like $A$ values: spin-forbidden E1 and allowed transitions between the $2 s^{2} 2 p^{4}$ and $2 \mathrm{~s} 2 \mathrm{p}^{5}$ configurations, and between the $2 \mathrm{~s} 2 \mathrm{p}^{5}$ and $2 \mathrm{p}^{6}$ configurations (last two transitions) for S IX. 24C: Present calculation; 6C: 6-configuration calculation described in Bhatia \& Landi (2003b); F86: Fawcett (1986); ZS02: Zhang \& Sampson (2002); TFF02: Tachiev \& Froese Fischer (2002); C79: Cheng et al. (1979).

\begin{tabular}{rrrrrrr}
\hline \hline Transition & $24 \mathrm{C}$ & $6 \mathrm{C}$ & $\mathrm{F} 86$ & ZS02 & TFF02 & C79 \\
\hline${ }^{3} \mathrm{P}_{2}-{ }^{3} \mathrm{P}_{2}$ & $1.332+10$ & $1.494+10$ & $1.420+10$ & $1.571+10$ & $1.248+10$ & $1.642+10$ \\
${ }^{3} \mathrm{P}_{2}-{ }^{3} \mathrm{P}_{1}$ & $7.845+09$ & $8.796+09$ & $8.348+09$ & $9.284+09$ & $7.361+09$ & $9.685+09$ \\
${ }^{3} \mathrm{P}_{2}-{ }^{1} \mathrm{P}_{1}$ & $4.043+08$ & $4.911+08$ & & $4.723+08$ & $4.092+08$ & $5.648+08$ \\
${ }^{3} \mathrm{P}_{1}-{ }^{3} \mathrm{P}_{2}$ & $4.225+09$ & $4.744+09$ & $4.537+09$ & $4.988+09$ & $3.955+09$ & $5.219+09$ \\
${ }^{3} \mathrm{P}_{1}-{ }^{3} \mathrm{P}_{1}$ & $4.436+09$ & $4.979+09$ & $4.735+09$ & $5.244+09$ & $4.157+09$ & $5.477+09$ \\
${ }^{3} \mathrm{P}_{1}-{ }^{3} \mathrm{P}_{0}$ & $1.825+10$ & $2.048+10$ & $1.952+10$ & $2.163+10$ & $1.712+10$ & $2.254+10$ \\
${ }^{3} \mathrm{P}_{1}-{ }^{1} \mathrm{P}_{1}$ & $1.077+07$ & $1.235+07$ & & $1.171+07$ & $1.052+07$ & $4.434+07$ \\
${ }^{3} \mathrm{P}_{0}-{ }^{3} \mathrm{P}_{1}$ & $5.778+09$ & $6.485+09$ & $6.194+09$ & $6.839+09$ & $5.417+09$ & $7.127+09$ \\
${ }^{3} \mathrm{P}_{0}-{ }^{1} \mathrm{P}_{1}$ & $2.318+07$ & $2.681+07$ & & $3.193+07$ & $2.209+07$ & $3.507+07$ \\
${ }^{1} \mathrm{D}_{2}-{ }^{3} \mathrm{P}_{2}$ & $6.198+07$ & $7.161+07$ & & $7.058+07$ & $5.973+07$ & $8.287+07$ \\
${ }^{1} \mathrm{D}_{2}-{ }^{3} \mathrm{P}_{1}$ & $3.148+06$ & $3.375+06$ & & $5.391+06$ & $3.156+06$ & $6.343+06$ \\
${ }^{1} \mathrm{D}_{2}-{ }^{1} \mathrm{P}_{1}$ & $6.005+10$ & $7.133+10$ & $6.340+10$ & $7.017+10$ & $5.736+10$ & $7.521+10$ \\
${ }^{1} \mathrm{~S}_{0}-{ }^{3} \mathrm{P}_{1}$ & $1.199+07$ & $1.711+07$ & & $1.559+07$ & $1.048+07$ & $1.847+07$ \\
${ }^{1} \mathrm{~S}_{0}-{ }^{1} \mathrm{P}_{1}$ & $4.062+09$ & $4.549+09$ & $4.761+09$ & $4.863+09$ & $3.967+09$ & $5.385+09$ \\
${ }^{3} \mathrm{P}_{1}-{ }^{1} \mathrm{~S}_{0}$ & $1.243+08$ & $1.695+08$ & & $1.430+08$ & $1.249+08$ & $1.760+08$ \\
${ }^{1} \mathrm{P}_{1}-{ }^{1} \mathrm{~S}_{0}$ & $5.181+10$ & $7.240+10$ & $5.467+10$ & $6.553+10$ & $4.868+10$ & $7.274+10$ \\
\hline
\end{tabular}

Cheng et al. (1979) overestimate the $A$ value; the agreement for the 5-9 transition is worse $(25-35 \%)$ than for the 4-9 one, but again better agreement is found with results obtained in the present work, Tachiev \& Froese Fischer (2002) and Vilkas et al. (1994).

\section{Conclusions}

In the present work we have calculated a complete, selfconsistent dataset of atomic data and transition probabilities for all the most abundant ions in the O-like sequence with $Z$ included between 11 and 30. The main aim of the present work is to develop complete sets of radiative data $(A$ values and oscillator strengths) for the less-abundant elements $(\mathrm{P}, \mathrm{Cl}, \mathrm{K}$,
Ti, Cr, Mn, Co), unavailable in the literature. Results have been compared with earlier calculations, where available; energy levels have been found to be slightly less accurate than previous work for the lighter ions, and in very good agreement for the heavier ions. Despite this, the accuracy of the $A$ values and oscillator strengths, the main topic of the present work, is comparable to, or better than, all the most sophysticated calculations available in the literature. A comparison with Si VII oscillator strengths measured in the laboratory confirms the accuracy of the radiative values of the present calculation.

This dataset is the first one to provide complete radiative data for all the elements considered, and fills a few gaps for the 
Table 11. Comparison between different calculations of O-like $A$ values: spin-forbidden E1 and allowed transitions between the $2 s^{2} 2 p^{4}$ and $2 \mathrm{~s} 2 \mathrm{p}^{5}$ configurations, and between the $2 \mathrm{~s} 2 \mathrm{p}^{5}$ and $2 \mathrm{p}^{6}$ configurations (last two transitions) for Fe XIX. 24C: Present calculation; BZ01: Butler \& Zeippen (2001); L85: Lolergue et al. (1985); ZS02: Zhang \& Sampson (2002); F86: Fawcett (1986); V94: Vilkas et al. (1994); C79: Cheng et al. (1979).

\begin{tabular}{rrrrrrrr}
\hline \hline Transition & $24 \mathrm{C}$ & BZ01 & L85 & ZS02 & F86 & V94 & C79 \\
\hline${ }^{3} \mathrm{P}_{2}-{ }^{3} \mathrm{P}_{2}$ & $3.492+10$ & $3.530+10$ & $3.910+10$ & $3.867+10$ & $4.585+10$ & $3.349+10$ & $3.923+10$ \\
${ }^{3} \mathrm{P}_{2}-{ }^{3} \mathrm{P}_{1}$ & $2.855+10$ & $2.900+10$ & $3.180+10$ & $3.146+10$ & $2.949+10$ & $2.728+10$ & $3.223+10$ \\
${ }^{3} \mathrm{P}_{2}-{ }^{1} \mathrm{P}_{1}$ & $1.083+10$ & $1.130+10$ & $1.140+10$ & $1.237+10$ & $1.213+10$ & $1.032+10$ & $1.314+10$ \\
${ }^{3} \mathrm{P}_{0}-{ }^{3} \mathrm{P}_{1}$ & $1.451+10$ & $1.480+10$ & $1.640+10$ & $1.594+10$ & $1.528+10$ & $1.402+10$ & $1.619+10$ \\
${ }^{3} \mathrm{P}_{0}-{ }^{1} \mathrm{P}_{1}$ & $1.281+09$ & $1.310+09$ & $1.110+09$ & $1.516+09$ & & & $1.626+09$ \\
${ }^{3} \mathrm{P}_{1}-{ }^{3} \mathrm{P}_{2}$ & $9.383+09$ & $9.530+09$ & $1.060+10$ & $1.036+10$ & $9.828+09$ & & $1.060+10$ \\
${ }^{3} \mathrm{P}_{1}-{ }^{3} \mathrm{P}_{1}$ & $1.133+10$ & $1.150+10$ & $1.270+10$ & $1.256+10$ & $1.176+10$ & $1.092+10$ & $1.283+10$ \\
${ }^{3} \mathrm{P}_{1}-{ }^{3} \mathrm{P}_{0}$ & $5.445+10$ & $5.520+10$ & $6.070+10$ & $6.034+10$ & $5.657+10$ & $5.218+10$ & $6.160+10$ \\
${ }^{3} \mathrm{P}_{1}-{ }^{1} \mathrm{P}_{1}$ & $8.436+08$ & $8.820+08$ & $9.510+08$ & $8.902+08$ & & & $1.003+09$ \\
${ }^{1} \mathrm{D}_{2}-{ }^{3} \mathrm{P}_{2}$ & $1.935+09$ & $2.040+09$ & $2.170+09$ & $2.142+09$ & $2.201+09$ & $1.887+09$ & $2.286+09$ \\
${ }^{1} \mathrm{D}_{2}-{ }^{3} \mathrm{P}_{1}$ & $1.685+07$ & $1.690+07$ & $2.780+07$ & $3.166+06$ & & $2.429+07$ & $5.150+06$ \\
${ }^{1} \mathrm{D}_{2}-{ }^{1} \mathrm{P}_{1}$ & $1.363+11$ & $1.380+11$ & $1.500+11$ & $1.502+11$ & $1.401+11$ & $1.317+11$ & $1.544+11$ \\
${ }^{1} \mathrm{~S}_{0}-{ }^{3} \mathrm{P}_{1}$ & $6.364+08$ & $6.610+08$ & $6.520+08$ & $7.628+08$ & & $5.864+08$ & $8.227+08$ \\
${ }^{1} \mathrm{~S}_{0}-{ }^{1} \mathrm{P}_{1}$ & $9.798+09$ & $9.890+09$ & $1.080+10$ & $1.058+10$ & $1.105+10$ & $9.748+09$ & $1.116+10$ \\
${ }^{3} \mathrm{P}_{1}-{ }^{1} \mathrm{~S}_{0}$ & $1.026+10$ & $1.070+10$ & $1.170+10$ & $1.110+10$ & & $9.834+09$ & $1.249+10$ \\
${ }^{1} \mathrm{P}_{1}-{ }^{1} \mathrm{~S}_{0}$ & $1.403+11$ & $1.420+11$ & $1.590+11$ & $1.602+11$ & $1.432+11$ & $1.354+11$ & $1.681+11$ \\
\hline
\end{tabular}

Table 12. Comparison of measured and calculated $A$ values for the 4-9 and 5-9 transitions in Si VII. Wavelengths are calculated from observed energy levels from Edlen (1983). The measured $A$ value was obtained from the absorption oscillator strength measured by Flaig $\&$ Schartner (1985) using the wavelengths listed.

\begin{tabular}{|c|c|c|c|c|c|c|c|c|}
\hline Transition & Wvl. (Å) & $f_{\mathrm{obs}}$ & $A_{\mathrm{obs}}$ & $24 \mathrm{C}$ & TFF02 & V94 & ZS02 & C79 \\
\hline${ }^{1} \mathrm{D}_{2}-{ }^{1} \mathrm{P}_{1}$ & 217.83 & 0.149 & $3.49+10$ & $4.72+10$ & $4.38+10$ & $4.35+10$ & $5.62+10$ & $6.12+10$ \\
\hline${ }^{1} \mathrm{~S}_{0}-{ }^{1} \mathrm{P}_{1}$ & 246.12 & 0.085 & $3.12+9$ & $3.01+9$ & $2.90+9$ & $2.92+9$ & $3.82+9$ & $4.30+9$ \\
\hline
\end{tabular}

less abundant ions (P, Cl, K, Ti, Cr, Mn, Co). The present data will be included in the next release of the CHIANTI database.

Acknowledgements. The work of the author is supported by the NNH04AA12I, W10,232 and NNG04ED07P NASA grants. The author wishes to thank Dr. A. K. Bhatia (NASA/GSFC) and W. Eissner (Univ. Stuttgart, Germany) for very helpful discussions, and the anonymous referee for his/her valuable comments that helped improve the original manuscript.

\section{References}

Baluja, K. L., \& Zeippen, C. J. 1988, J. Phys. B, 21, 1455

Bhatia, A. K. 1982, J. Appl. Phys., 53, 59

Bhatia, A. K., Feldman, U., \& Doschek, G. A. 1979, A\&A, 80, 22

Bhatia, A. K., Feldman, U., \& Doschek, G. A. 1980, J. Appl. Phys., 51,1464

Bhatia, A. K., Landi, E., \& Mason, H. E. 2003a, ADNDT, 83, 71

Bhatia, A. K., Thomas, R. J., \& Landi, E. 2003b, ADNDT, 83, 113

Bhatia, A. K., \& Landi, E. 2003a, ApJ, 585, 587

Bhatia, A. K., \& Landi, E. 2003b, ADNDT, 85, 169

Butler, K., \& Zeippen, C. J. 2001, A\&A, 372, 1083

Cheng, K. T., Kim, Y.-K., \& Desclaux, J. P. 1979, ADNDT, 24, 111

Dere, K. P., Landi, E., Mason, H. E., Monsignori Fossi, B. C., \& Young, P. R. 1997, A\&ASS, 125, 149

Edlen, B. 1983, Phys. Scr., 28, 51
Eissner, W., Jones, M., \& Nussbaumer, H. 1974, Comput. Phys. Commun., 8, 270

Fawcett, B. C. 1986, Atom. Data Nucl. Data Tab., 34, 215

Feldman, U., Seely, J. F., \& Bhatia, A. K. 1985, Atom. Data Nucl. Data Tab., 32, 305

Feldman, U., Curdt, W., Landi, E., \& Wilhelm, K. 2000, ApJ, 544, 508

Feldman, U., Landi, E., \& Curdt, W. 2004, ApJ, 607, 103

Flaig, H. J., \& Schartner, K. H. 1985, Phys. Scr., 31, 255

Froese Fischer, C., \& Saha, H. P. 1983, Phys. Rev. A, 28, 3169

Froese Fischer, C., \& Tachiev, G. 2004, Atom. Data Nucl. Data Tab., 87,1

Galavis, M. E., Mendoza, C., \& Zeippen, C. J. 1997, A\&AS, 123, 159

Kink, I., Jupen, C., Engström, L., et al. 1997, ApJ, 487, 956

Kink, I., \& Engström, L. 1997, Phys. Scr., 56, 31

Loulergue, M., Mason, H. E., Nussbaumer, H., \& Storey 1985, A\&A, 150,246

Shirai, J., Musgrove, A., \& Wiese, W. L. 2000, J. Phys. Chem. Ref. Data, Monograph, 8

Tachiev, G., \& Froese Fischer, C. 2002, A\&A, 385, 716

Vilkas, M. J., Merkelis, G., Kiselius, R., et al. 1994, Phys. Scr., 49, 592

Young, P. R., Del Zanna, G., Landi, E., et al. 2003, ApJS, 144, 135

Zhang, H. L., \& Sampson, D. H. 2002, Atom. Data Nucl. Data Tab., 82,357 